\title{
PLANNING AND CONDUCTING COMPUTER ASSISTED EXERCISES DURING A PANDEMIC
}

\author{
Diana-Ioana ZINCA \\ Multinational Division South-East, Bucharest, Romania \\ zinca_diana@yahoo.com \\ Ghiță BÂRSAN \\ "Nicolae Bălcescu" Land Forces Academy, Sibiu, Romania \\ ghbarsan@gmail.com
}

\begin{abstract}
2020 was a year that changed the world completely, forced everyone to think outside of the box and the effects, as well as the results, are still to be measured. While facing several countries' lockdowns, planning and conducting a multinational computer assisted exercise (CAX) during a pandemic seemed nearly impossible. By taking extreme measures, developing a new architecture model for a CAX and relying on the training audience's will to be trained, NATO managed to overcome all the constraints and successfully conducted the exercise Loyal Leda 2020. This paper will not only give details about the new CAX architecture concept that supported the execution phase of the exercise, but will also explain the exercise planning process (EPP) behind it.
\end{abstract}

KEYWORDS: computer assisted exercise, distributed exercise control, simulation systems

\section{Introduction}

Training represents an important part of the military domain. For a soldier on duty within a theater of operation, having proper training will save his life, while for the commander who works at the tactical level, having proper training translates into making the right decisions to complete the mission while keeping his subordinates alive in the process. Things become even more complicated when it comes to providing training for the higher headquarters' commanders working at the operational or strategic levels. To achieve that, the North Atlantic Treaty Organization (NATO) developed two main areas of interest: the Individual domain- comprising of education and individual training and the Collective area, which hosts the collective training and the exercises (NATO Allied Command Transformation, 2013). "The focus of NATO's Collective Training and Exercise (CT\&E) programme is to ensure that the Alliance has a coherent set of deployable, interoperable and sustainable forces that are equipped, trained, exercised, and commanded so as to meet NATO's Level of Ambition (LoA)" (NATO Allied Command Transformation, 2020). The best way to train those attributes is through major joint exercises (North Atlantic Military Committee, 2014).

Based on how the exercise is delivered to the training audience (TA) and how it is controlled, the exercises can be divided into different types. The most used 
in NATO are the computer assisted exercises (CAX). Within a CAX modelling and simulation systems and techniques are used to replicate a real-world environment that will stimulate and support the command and control actions, as well as the decision making process. Specific scenarios are used to provide the TA with the opportunity to conduct Major Joint Operations (MJOs) and to reach their training objectives (TO). The complexity of the exercise architecture is directly linked with the TA's level of ambition, therefore the planning process for a CAX can take up to 2 years. For a better understanding, we will briefly explain the CAX architecture and the exercise planning process according to the NATO doctrine and then we will focus on the exercise Loyal Leda 2020 and the distributed EXercise CONtrol (EXCON) concept that kept the exercise running, even during a pandemic.

\section{The CAX Structure}

When we speak about the CAX structure, we should think about two main entities that set the exercise architecture: the TA and the EXCON. To successfully run an exercise, a full and centralized control is needed in order to keep everyone on the same page and to guide the TA to reach their training objectives as well as the exercise objectives. A full control of the exercise is the reason why the agreed architecture for the NATO exercises looks like in the Figure no. 1.


Figure no. 1: CAX architecture based on the TA and EXCON locations (Source: Authors)

To better explain why this type of exercise architecture is agreed upon in NATO, first we have to highlight the differences between the TA and EXCON. The training audience is represented by the people who will be trained and evaluated (if that is the requirement) during the exercise. There are two categories of TA: primary training audience (PTA - those that are usually evaluated, therefore, they are the exercise's main focus) and the secondary training audience (STA - who also have some TOs to achieve during training, as long as they do not interfere with the PTA requirements). In terms of the systems they use during the exercise, everything should be like in real life. Meaning that all the command and control systems (functional area services - FASs) that they are using in their daily activities will be used during the 
exercise as well (Bârsan \& Zinca, 2018). As exercise planners, our goal is to provide the TA with a simulated environment and scenario during the exercise, as close to reality as possible. In order to fulfill our task, the simulation systems and the EXCON tools come in our support. Those systems actually create the main difference between the TA and the EXCON. If the TA can build their common operational picture (COP) on the FASs, based on the assets and the rights that they normally have, the EXCON has the "God view" due to the simulation systems (JTLS or JCATS) and their auxiliary tools like JEMM - Joint Exercise Management Module. The simulation system represents the ground truth, simulates the battle between the blue and red forces according with the plans and orders developed by the TA and also generates reports on behalf of the subordinate units / higher echelons (the Response Cells-RC, which are part of EXCON). The JEMM is the tool that helps EXCON to create additional incidents that trigger a certain reaction from the TA in order to achieve a training objective (Bârsan \& Zinca, 2020). JEMM tool is used mostly to support the unconventional threats within an exercise (since the conventional threats are mostly conducted in the simulation / game) (Ioniţă, Pînzariu, Bârsan, Raţiu \& Moşteanu, 2017).

Nowadays, the hybrid threats are plying a major role in the battlefield and being able to react properly, is must (Badea, Bârsan, Moşteanu, Iancu, 2016). Speaking of the main differences between the TA and EXCON, last but not least, we have to mention that the EXCON knows the red forces campaign plan in detail. Based on the given scenario, the TA's Intel branches develop the enemy most likely and the most dangerous courses of actions (CoAs) during the operational planning process (OPP). Those are based on the information that they have available through the exercise scenario and the assessments that they make. Based on the enemy CoAs, during the OPP the TA develops their campaign plan/ scheme of maneuver. The red forces campaign is developed during the exercise planning process (EPP) by the EXCON cell called opposing forces (OPFOR). Usually the OPFOR cell comprises of subject matter experts (SMEs) from the training center and the training audience (trusted agents). The red force's campaign, which is developed by the EXCON, and the blue force's campaign (and the enemy CoA), which is developed by the TA, should be similar/ make sense (if not, both the TA and the OPFOR will make adjustments in order to be on the same page). This does not mean that the TA knows what will happen. They know the enemy intention, his goals, assets and the way he fights according to his doctrine.

There is one important thing to keep in mind: the EXCON develops an exercise to challenge all the TA's domains in order for them to be properly trained (to reach their TOs). This means that both the red force's campaign and the Main Even List/ Main Incident List (MEL/MIL) database in JEMM are directly linked with the TA TOs and the EXCON main job during the exercise is to keep the TA on track in order to face all the scripted problems and to overcome them. This is actually the main issue for EXCON: to force the TA to meet their TOs when all that they want is to win the war. This is the reason why NATO agreed to use the exercise architecture depicted in Figure no. 1 in the first place: to have the EXCON structure in one place, under Officer Directing the Exercise (ODE) command, to fully control the exercise and to be able to guide the TA. Based on the exercise complexity, the EXCON might reach hundreds of people, operating not only on EXCON systems, but also on the functional area services. You can see in Figure no. 2 how the EXCON is structured. 


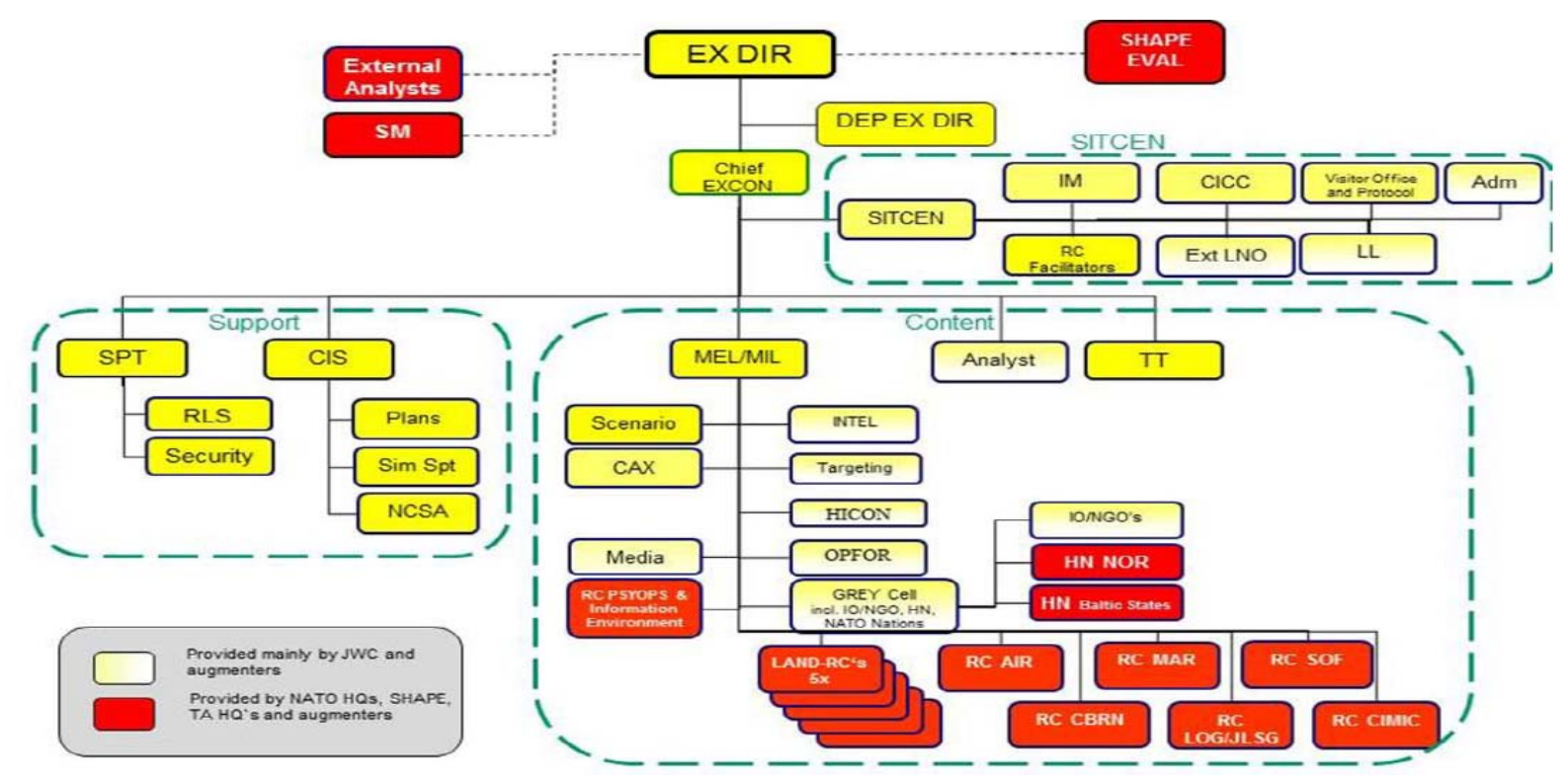

Figure no. 2: Example of an EXCON structure for an exercise

(Source: NATO Allied Command Transformation, 2020)

The EXCON personnel have a complex understanding of the exercise scenario, red and blue forces' campaigns, training objectives, their interaction with the TA and the role that they are playing. Therefore, the most difficult role in an exercise is played by the EXCON team (especially RCs), not the TA.

As depicted in Figure no. 1, the training audience can be located at their home bases or in the same area as EXCON, not in the same place though. During the exercise there is no physical interaction between the EXCON and the TA. To constantly track TA's actions, the exercise design has trusted agents to play an important role. They are part of EXCON, are co-located with the TA, they have access to some of the EXCON tools (like JEMM) and are in permanent connection with the EXCON cell. Their job is to monitor the TA's actions, to guide them and to keep them on track.

Bottom-line, to have an exercise up and running, we need to have the TA in place (either deployed on the field, in their home bases or at the training center) and the EXCON in one single place, with trusted agents to monitor the TA performance.
Now imagine that you have to run a NATO exercise while facing a pandemic which forces international lock-down for many countries, with travel restrictions and social distancing in place and restrictions regarding the number of people that are allowed to be in one room. The biggest problem? - how to build and coordinate the EXCON. No EXCON, no exercise.

\section{Loyal Leda 2020 (LOLE20) - Distributed EXCON (DEXCON)}

The exercise LOLE20 was the last part of the Trident Jupiter 2019 (TRJU) series. It was an exercise which certified Allied Rapid Reaction Corp (ARRC) as a NATO War Fighting Corp Headquarters. Taking into consideration the fact that the exercise TRJU19 part 2 was supposed to be played at the end of March 2020 and was cancelled on short notice (a couple of weeks before the execution phase), LOLE20 which was planned to be executed in November couldn't afford to follow the same path and also be cancelled due to the second wave of COVID-19 pandemic. Therefore, during the EPP conferences, several contingency plans were discussed. All of them implied EXCON personnel to 
travel to the training center though. We briefed as well the concept of distributed EXCON, but since it had never been done before in NATO and we were far into the EPP, changing the exercise architecture at that point seemed too risky for the exercise planning team. One of the contingency plans developed was regarding the minimum training requirements (MTRs) that the TA should reach in order to become certified. The task was for the TA to establish those MTRs together with the Combat Readiness Evaluation (CREVAL) team in order for the exercise team to be able to quickly readjust the JEMM database and be able to support the exercise with a smaller EXCON (if needed) and without having an impact on the TAs' evaluation and certification.

The exercise LOLE20 was developed as a multi-level exercise, meaning that it had multiple TAs, on different levels. ARRC was the PTA and as STA was established Multinational Division SouthEast Headquarters (MND-SE). The exercise was supposed to be conducted in 3 different countries (Bucharest, Romania for MND-SE, Bydgoszcz, Poland for EXCON and a location in the UK or Germany where ARRC was supposed to deploy). That was the planned exercise architecture to be played, no different than normal as depicted in Figure no. 1. We have to highlight the fact that parts of EXCON were the Canadian, US and UK Divisions, as well as a Corp from Italy - personnel from all of them were supposed to travel to the training center in Poland. Everything seemed to be ready for the execution phase of the exercise, but the second wave of COVID19 struck Europe in mid-October, followed by more restrictions and lock-downs.

\subsection{Distributed EXCON Concept (DEXCON)}

Facing the problem that no one wanted to consider during the EPP conferences - a lock-down due to the pandemic second wave - forced all the officers with primary responsibility (OPRs) to think outside of the box and come up with another contingency plan. Now, DEXCON concept seemed a viable solution to keep the exercise alive, even if it was something that had never been done in NATO, and was extremely hard for the EXCON team to coordinate and implied changing the exercise architecture on a short notice. An exercise based on DEXCON architecture is exampled in Figure no. 3.

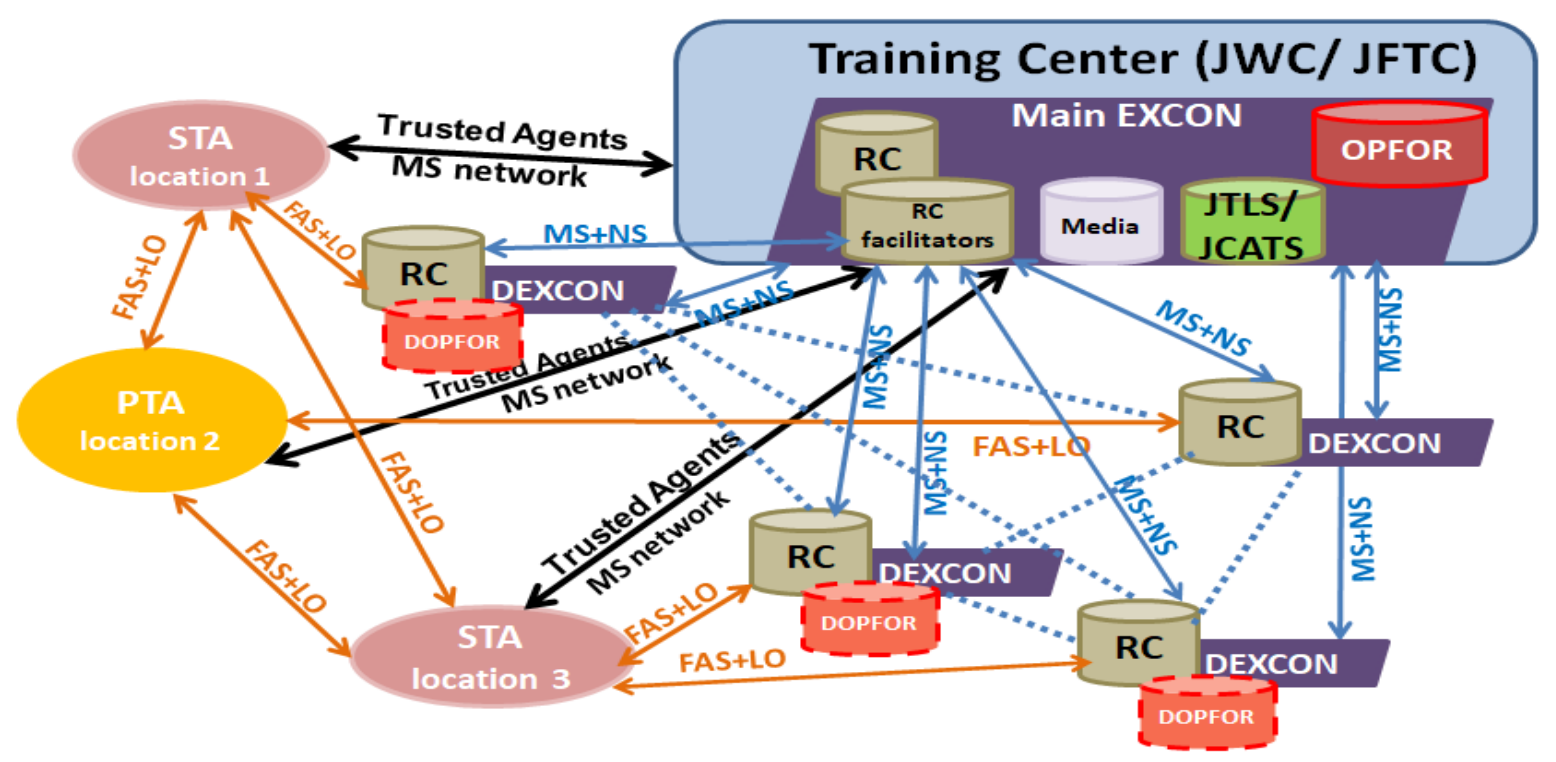

Figure no. 3: $D E X C O N$ architecture for a $C A X$

(Source: Authors) 
There are several factors that have to be taken into consideration before planning an exercise with a DEXCON. First is the CIS problem. All the systems should be interconnected (FASs and simulation systems). This narrows the pool for the EXCON. If the TA is a NATO HQ (since we are speaking about a NATO exercise), the STA or the RCs (affiliated units) are not always NATO HQs, but national units. The problem is that the national CIS systems are not able to feed the NATO systems or the other way around. Technical solutions might be found, such as various bridges, but the reality is that the more we rely on CIS and networks that are not 100 $\%$ interconnected, the more we risk a CIS failure during the execution phase, which will jeopardize the whole exercise. If we can afford finding technical solutions for a STA to feed the COP for the PTA ("train as you fight"), the EXCON should be working on the same systems in order to control the exercise. Keep in mind that "train as you fight" only applies to the TA, not the EXCON. The EXCON are the people who create the story of the conflict for the TA to fight and train. In a real life conflict / crisis situations, there is no EXCON. Therefore, there is no need to train the EXCON and put pressure on them because they have their hands full by trying to provide the training opportunity for the TA as well as evaluating them. In crisis situations, we are not speaking about RCs or EXCON anymore. In a conflict, the subordinate units would play the same role as the higher headquarters, all of them being "TA". There will be no simulation system (controlled by the EXCON) running the fight and reporting on time based on a mathematical model (that is based usually on Lanchester's equations). The artificial environment regarding the subordinates units reporting on time, usually with all the information needed, the COP updated without major issues that we provide during an exercise to the TA is far from being realistic. It is a compromise made in order for the TA to achieve all their training objectives in 10 days - which are not CIS related (there are different exercises focused on testing the CIS, but are not part of this research paper). Keep in mind that the EXCON is committed to provide the realistic environment for the TA to "train as they fight" in order to reach the specified exercise training objectives. The reason why the TOs are extremely important in an exercise is because the whole architecture of the exercise is designed around them. As an exercise planner, once you have the TOs, you know the parts that you need to focus on in order to provide the proper training. In 10 days you cannot train all the domains, you cannot perfectly replicate the conflict and you certainly cannot win the war. The different CIS networks (NATO and nationals) were the main reason why we had to adjust the DEXCON concept for the exercise LOLE20 in order to make it work. The DEXCON concept with a customed structure for LOLE20 will be further explained in the next subsection.

Apart from the CIS systems, in order to successfully run a DEXCON exercise, teams of SMEs are needed in all the locations of the distributed EXCONs. As shown in Figure no. 3, the main EXCON would be run at the simulation center, by their SMEs and in each location of the RCs would be a smaller team of DEXCONs SMEs, capable to fully controlling the RCs that they are in charge of. All the DEXCONs are subordinate to the main EXCON, which is driven by the EXercise DIRector (EXDIR). Aquiring the right personnel from all locations, with the right expertise for the DEXCONs jobs is far from easy. The minimum functions that a DEXCON should have are:

DEXCON Chief - a senior officer with tactical and operational level expertise, as well as exercise planning knowledge and a detailed comprehension of the exercise scenario and the campaign plans. 
He / she will lead the DEXCON team, will be the main point of contact for the main EXCON and will work closely with the RCs Chiefs and the trusted agents from the TA (or the Liaison Officer);

MEL / MIL SME - preferably the exercise OPR or someone from the core planning team who attended the MEL / MIL workshops during the EPP, with further knowledge of the exercise scenario, all the storylines developed for the exercise, the campaign plans and detailed knowledge in working with JEMM. He/she will lead the team for the MEL / MIL dynamic scripting for the RCs, will be responsible for all the storylines and injects to be carried out on time and will be part of all the MEL / MIL EXCON coordination working groups with the main EXCON. $\mathrm{He} /$ She can act as the deputy of Chief DEXCON if needed and will lead the EXCON briefings for their RC, during the EXCON training, prior to the STARTEX;

CAX SME - is a person with professional training on the simulation system used during the exercise (JCATS or JTLS). He / She is preferably also a CAX operator (for aviation assets or artillery) and will be responsible for supporting the CAX operators with additional training if required, will brief the RCs regarding the capabilities of the simulation system, its role and how it is related to the FASs and will also be the point of contact for the CAX team in the main EXCON. He / She will work closely with the RC members and the MEL / MIL DEXCON SME for the MEL / MIL database validation and for scripting the action injects when needed;

OPFOR SME - is the person with a detailed knowledge about the OPFOR campaign and will be especially responsible to track the red forces from the area of operation of his / her RC. He / She will work in close coordination with the MEL / MIL DEXCON SME and the RC members in order to validate the JEMM database and to help scripting the action injects for the OPFOR. He / she will constantly be part of the OPFOR working groups held by the main EXCON. If it is decided to also distribute the OPFOR, he will be in charge of the DOPFOR team and will be responsible of all the red units' missions that are allocated to him / her (including operating them in the simulation system);

Last but not least, it is highly important to have a RC chief with a solid background in both operational and exercise planning and to include the entire core planning team who attended the EPP conferences (including the ones who participated at the MEL/MIL workshops) as part of the RC.

As we stated before, being EXCON is the hardest job that you can have during an exercise. Moreover, being part of the RC is even more complicated because as a $\mathrm{RC}$ you are double-headed. You have two entities that you are subordinated to: the EXCON (or DEXCON) from the exercise perspective and your higher HQ (the TA) from the operational point of view. As part of EXCON, you know what will happen, but you report and you brief the TA only with the things that they are allowed to know. This is the reason why it is extremely important that all the RC personnel understand the difference between being TA versus being a RC.

If the proper interconnected CIS systems, the SMEs to run the main EXCON, as well as the DEXCONs and the RCs are had, this type of distributed architecture can be applied to run the exercises from different locations and still be able to properly support the TA and their requirements. DEXCON concept (with one main EXCON and 2 DEXCONs) was successfully applied for the first time in NATO during the exercise LOLE20 and proved that where there is a will for training, there is a way. 


\subsection{LOLE20 Execution Phase}

LOLE20 EPP started in 2018, based on the architecture depicted in Figure no. 1. At the beginning of the EPP, the exercise was supposed to have ARRC as PTA and two Divisions as STAs. After the Main Planning Conference in March 2020, it was established that only ARRC will remain as PTA and MND-SE as STA. Back then the pandemic had just started in Europe and the TRJU 19-2 exercise was about to be cancelled. Being fairly advanced in the EPP, the pandemic didn't impact the planning much. Of course, it made things way more difficult in terms of training (Academics were conducted online, the Battle Staff Training was conducted at a smaller scale, using other networks than the one for the exercise), and conferences. Some core planning team meetings (CPTM) and the Final Coordination Conference were held online. The real challenge was the MEL / MIL Strategy and Scripting workshops (WS) which could not be fully conducted online. By September, the first COVID wave calmed down slightly and allowed the MEL / MIL WSs to take place in a hybrid manner (both in person and online). At the same time the CPTM-3 was conducted with the MEL / MIL WSs which allowed the OPRs to start developing contingency plans for the execution phase of the exercise in detail. As we described before, due to the fact that the pandemic was slowing down, travelling was not a huge issue anymore, the focus of the plan was to keep LOLE20 up and running and providing ARRC the minimum training requirements in case the COVID cases rose again and we had to shrink the number of personnel from the EXCON. At that point it was established that ARRC will deploy at Royal Air Force South Cerney and Fairford in UK as PTA, while MND-SE will play from Bucharest, Romania as STA. The EXCON (and all the RCs from US, UK, Romania and Canada) were supposed to deploy for the execution phase at the Joint Force Training Center (JFTC) in Poland (Porcile, 2003). All the CIS architecture (mission secret network) (Zima, 2020) was established accordingly in all the above mentioned locations. Three weeks before the execution phase, the second wave of COVID-19 struck again and travel restrictions were in place in Poland- no one could travel there anymore. Starting from the DEXCON concept, JFTC as ODE, the Officer Scheduling the Exercise (OSE), Officer Conducting the Exercise (OCE), together with the CIS SMEs and the OPRs from each structure developed several courses of actions for LOLE20. The agreed upon action is depicted in Figure no. 4.

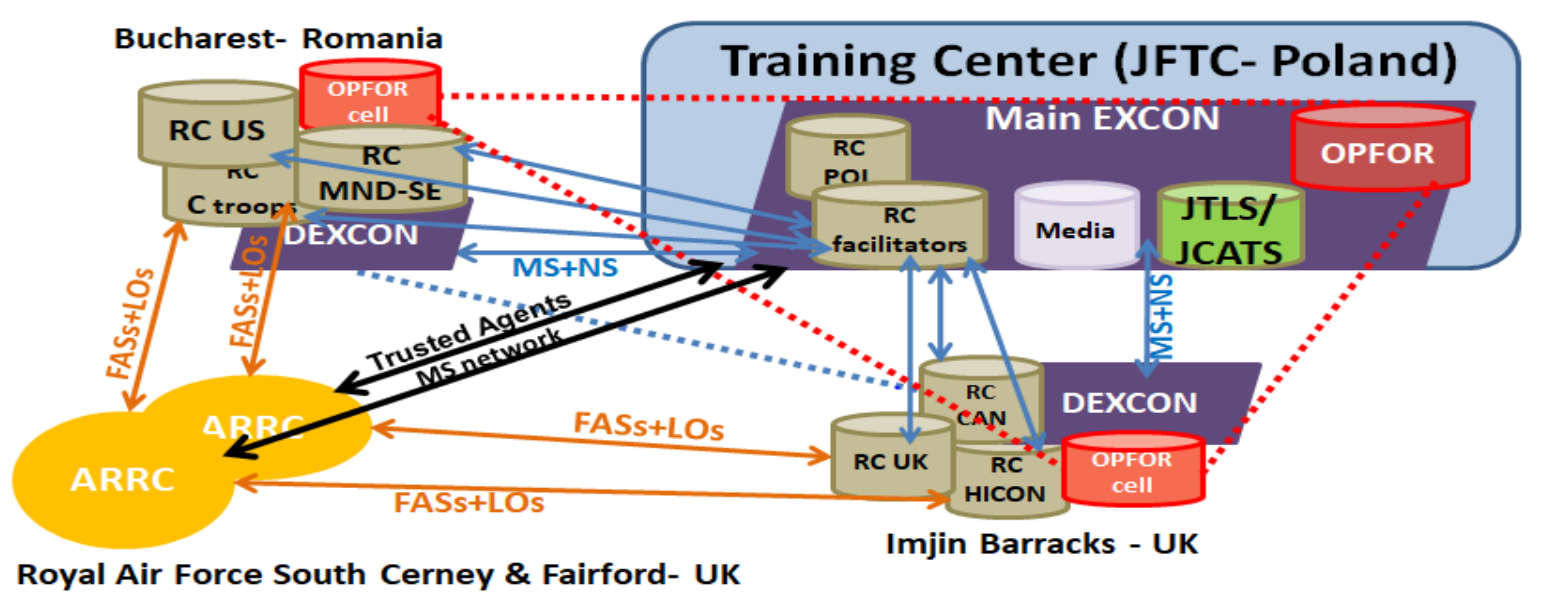

Figure no. 4: LOLE20 execution phase architecture

(Source: Authors) 
The reason why we say that DEXCON concept was used at a lower scale for LOLE20 is due to the incompatibilities between the nationals and the NATO CIS networks (previously explained), the exercise was not possible to be distributed to all the locations where the RCs were supposed to be (Canada, US, Romania and UK). Therefore, a mitigation solution was needed: the entire CIS infrastructure for the exercise would be used at its full capacity in all three locations; the MTRs would be in place; MND-SE turned from a STA into a RC and all the other RCs would travel either to Bucharest or to the UK in order to support the exercise. That being said the main EXCON was in Poland at JFTC and 2x DEXCONs were built, one in Bucharest and the other one at Imjin Barracks in the UK. The Canadian and the UK Divisions, as well as the representatives from Italy for the higher HQ Corp, traveled to the UK and played their role as RCs, while Bucharest hosted MND-SE, the US Division and representatives from the ARRC troops. For better control, the simulation was not distributed at the DEXCONs locations. The CAX team from JFTC came up with the idea of remote CAX, meaning that the CAX operators from the RCs had access on the Joint Theater Level Simulation (JTLS) Web-Hosted Interface Program (WHIP) and were able to operate it remotely, using the same server. JFTC had full control on JTLS, meaning that the CAX team from JFTC was responsible for constantly updating the COP. Based on the exercise architecture, it was agreed that the simulation system would automatically feed the functional area services (the COP), based on the assets and capabilities that each unit had. The CAX operators from the RCs were putting orders into JTLS and JTLS was pushing the image into the FASs in order for the TA to be able to see / track the COP.

Moreover, another thing that secured LOLE20's success was the RC facilitators from JFTC. There were the DEXCONs teams which had counter-partners in the main EXCON, but there were also RCs representatives for each Division in the Main EXCON. They were constantly in contact with the RC Chief and the DEXCONs, updating and guiding them all the time. Last but not least, in order to make sure that the EXCON was able to coordinate with DEXCONs, both mission secret and the NATO secret networks were used.

LOLE20 was conducted between $9-19^{\text {th }}$ of November 2020 in three countries, with the participation of over 1000 personnel, and allowed ARRC to take its CREVAL and to become certified as NATO's War Fighting Corp HQ. The exercise LOLE20 not only certified ARRC, but also validated the DEXCON concept and proved that it can be a real alternative solution for conducting future NATO exercises.

\section{Conclusions}

Collective training implies people working closely together. After all this is the whole idea of it - to train people to be experts in their domains and to develop their team working skills. This idea applies for military exercises as well. In NATO, individual training is responsible for providing the proper knowledge for the personnel (through courses, etc.), while collective training is focused on providing all the opportunity to apply their knowledge and work together through the same goal: mission achievement.

The pandemic changed our world and the physical distance switched our way of living. In order to be safe, nowadays you need to keep distance from other people, meaning that the online environment turned into the new reality. When it comes to training, this cannot be fully applicable. If we can attend online courses without major problems, the collective training domain was badly impacted by the pandemic. At the tactical level, live or 
virtual simulation systems to train small units can still be used. When it comes to training Divisions, Corps HQs or units at the operational or strategic level, keeping social distance and facing lock-downs make training almost impossible.

The DEXCON concept, as it was proved during LOLE20, is a viable solution for supporting multinational exercises. We are confident that with the proper CIS support and the SMEs needed in EXCON and DEXCONs structures, it is possible to run a distributed major NATO exercise. This does not mean that this new concept should change NATO exercises architecture from now on. It is important to be able to run them, meaning that some NATO exercises based on the distributed architecture structure are certainly needed in order to find the optimal solutions. It is very important to have the comfort that no matter what happens, NATO is able to adjust and provide the required training in order to maintain the Alliance's level of security. Being able to run, coordinate and control an exercise from different locations speaks greatly about the performance of NATO CIS systems. Nevertheless, if a solution is not found to make all the national and CIS systems able to speak with each other, a distributed exercise makes no difference for the TA. The only difference is at the EXCON level, and as we stated before, it is nice to have SMEs able to run DEXCONs, nevertheless the experience and knowledge that they gain after a distributed exercise can be applied only in the training domain. The MEL/MIL WSs are part of the EPP and the first rule that is briefed before developing the storylines is: "No EXCON/ RC to RC play". Meaning that everything that is scripted during the WS should affect only the TA, not the RCs/
EXCON. A distributed exercise without making the national and NATO CIS systems interconnected puts a lot of pressure on the EXCON team, needs further investment and becomes harder to control the exercise. As you can see, there is no benefit for the TAs and the focus of the exercise is the TA, not the EXCON. Having the CIS systems interconnected though, would make a lot of difference and would be a good reason making worth the effort of running a distributed exercise. The reason why we say that is because once the technical solution for the systems is found, it will bring value for the "train as you fight" concept. The NATO HQs which play the TA will be able to use the NATO command and control systems and FASs, while getting reports from the subordinate units (the affiliated units) from their national CIS systems. That will bring a lot of realism to the exercises in terms of reports and returns and building the COP. Taking into consideration the costs, most likely it would be more feasible to have DEXCONs in the RCs locations and stretch the NATO CIS architecture a little for the EXCON, rather than moving all the RCs with their nationals CIS systems to one location.

To sum up, we do believe that it is very important for the DEXCON concept to be further developed and tested during other NATO exercises. We also think that a new procedure should be developed regarding the online training, for both individual and collective training. We are confident that the DEXCON concept works properly, nevertheless, taking into account the actual conditions and assets, we do not see this architecture as the main way to conduct an exercise, but as an alternate solution. 


\section{REFERENCES}

Badea, D., Bârsan, Gh., Moşteanu, D., Iancu, D. (2016). Landmarks of The Military Organisation's Approach as a Management Model. The $10^{\text {th }}$ International Management Conference, Management and Innovation for Competitive Advantage ICM, November 3-4, Romania, Bucharest: University of Economic Studies in partnership with Management Academic Society in Romania (SAMRO), ISSN 2286-1440, ISSN-L 2286-1440, 219-223.

Bârsan, Gh., \& Zinca, D. (2018). Constructive Simulation Programs and NATO Functional Area Services Applied in Computer Assisted Exercises. 161-165.

Bârsan, Gh., \& Zinca, D. (2020). Main Events List / Main Incidents List Development Process for Computer Assisted Exercises. International Conference Knowledge-Based Organization, Vol. 26, Issue 3, 146-150.

Ioniţă, D., Pînzariu, S., Bârsan, Gh. Raţiu, A., \& Moșteanu, D. (2017). Interinstitutional Dimension Concerning Planning, Training and Force Engagement as Response to The Hybrid War. Journal of Science of the Military Academy of Land Forces, Vol. 49, Issue 4(186), 38-48.

NATO Allied Command Transformation. (2013). Bi-Strategic Command Directive 075-002, Education and Training Directive. 13.

NATO Allied Command Transformation. (2020). Bi-Strategic Command Directive 075-003, Collective Training \& Exercise Directive. 11, 246.

North Atlantic Military Committee. (2014). NATO Education, Training, Exercises and Evaluation (ETEE) Policy. 14-18.

Porcile, C. (2003). Communication Information, available at: https://www.nato.int/nrdcit/magazine/2003/0307/03071.pdf.

Zima, A.J. (2020). NATO ARRC conducts exercise at RAF Fairford. U.S. European Command, available at: https://www.eucom.mil/article/40908/nato-arrc-conducts-exercise-atraf-fairford. 